\title{
Magnesium supplementation alleviates corticosteroid-associated muscle atrophy in rats
}

\author{
Lizhen Zheng ${ }^{1,2}\left(\mathbb{D} \cdot\right.$ Le Huang $^{1} \cdot$ Ziyi Chen $^{1} \cdot$ Can Cui $^{1} \cdot$ Ri Zhang $^{1} \cdot$ Ling Qin $^{1,3}$
}

Received: 20 December 2020 / Accepted: 21 May 2021 / Published online: 30 May 2021

c) Springer-Verlag GmbH Germany, part of Springer Nature 2021

\begin{abstract}
Purpose Corticosteroid (CS) therapy for infectious and rheumatological diseases showed to decrease serum magnesium $\left(\mathrm{Mg}^{++}\right)$level and induce muscle atrophy in patients. The present study investigated the effects of $\mathrm{Mg}^{++}$supplementation on preventing CS-induced muscle atrophy in an animal model, which provided experimental data for potential clinical translation.

Methods Twelve 24-week-old male Sprague-Dawley rats were treated with lipopolysaccharide (LPS) and CS methylprednisolone (MPS) to induce muscle atrophy, with half of the rats also given daily $50 \mathrm{mg} / \mathrm{kg} \mathrm{Mg}^{++}$oral supplementation. Additional six rats without LPS + CS treatments were used as normal controls. After treatment for 6 weeks, serum was collected for $\mathrm{Mg}^{++}$quantification, animal dual-energy X-ray absorptiometry (DXA) was performed for tissue composition, and the extensor digitorum longus (EDL) was collected for muscle functional test and histology including muscle fiber size, intramuscular fat infiltration and fiber typing. In vitro myotube atrophy model was used to study the in vitro effect associated with in vivo muscle atrophy.

Results LPS + CS treatments induced hypomagnesemia while the serum $\mathrm{Mg}^{++}$level was in normal range after $\mathrm{Mg}^{++}$supplementation. DXA showed 53.0\% lower fat percent and 29.7\% higher lean mass in LPS + CS + Mg group when compared to LPS + CS group. Muscle functional test showed 22.2\% higher specific twitch force and $40.3 \%$ higher specific tetanic force in LPS + CS + Mg group when compared to LPS + CS group. Histological analysis showed $4.1 \%$ higher proportion of muscle fibers area to total area and 63.6\% lower intramuscular fat infiltration in EDL sections in LPS + CS + Mg group when compared to LPS + CS group. LPS + CS + Mg group had 33.0\% higher area proportion and 29.4\% higher cross-sectional area (CSA) of type IIb muscle fiber. Myoblast culture results showed that $\mathrm{Mg}^{++}$supplementation group had larger myotube diameter. The mRNA expressions of the muscle atrophy marker genes MuRF1 and MAFbx were lower in $\mathrm{Mg}^{++}$supplementation group both in vitro and in vivo.

Conclusion The current study demonstrated that $\mathrm{Mg}^{++}$supplementation successfully alleviated CS-associated muscle atrophy in rats at both functional and morphology levels, indicating a translational potential for patients undergoing CS therapy. This study provided the evidence for the first time that $\mathrm{Mg}^{++}$supplementation could prevent muscle atrophy — an adverse effect of CS therapy, currently also adopted for treating coronavirus disease 2019 (COVID-19).
\end{abstract}

Keywords Corticosteroid $\cdot$ Magnesium $\cdot$ Muscle atrophy

Ling Qin

lingqin@cuhk.edu.hk

1 Musculoskeletal Research Laboratory of Department of Orthopaedics and Traumatology and Innovative Orthopaedic Biomaterial and Drug Translational Research Laboratory, Li Ka Shing Institute of Health Sciences, The Chinese University of Hong Kong, Rm74026, 5/F, Clinical Science Building, Prince of Wales Hospital, Shatin, Hong Kong SAR, People's Republic of China
2 Centre for Regenerative Medicine and Health, Hong Kong Institute of Science and Innovation, Chinese Academy of Sciences Limited, Hong Kong SAR, People's Republic of China

3 Hong Kong-Shenzhen Innovation and Technology Institute (Futian), The Chinese University of Hong Kong, Hong Kong SAR, People's Republic of China 


\section{Introduction}

Pulsed and long-term corticosteroid (CS) were frequently indicated in the treatment of infectious and rheumatoid diseases for modifying the disease progression and life-saving, such as Severe Acute Respiratory Syndrome (SARS), Systemic Lupus Erythematosus (SLE), and severe coronavirus disease 2019 (COVID-19) [1, 2]. A large clinical trial in the UK demonstrated that treatment with CS reduced onethird of deaths of patients critically ill with COVID-19 [3]. However, the side-effects of CS might include myopathy, electrolyte abnormalities, obesity, diabetes, osteoporosis, and osteonecrosis, etc., that were similar to Cushing's syndrome $[4,5]$.

CS-associated muscle atrophy was first described in 1932 in Cushing's syndrome [6] with an incidence of $60 \%$ in CStreated patients [7]. Recent studies showed increased muscular consequences in infectious diseases such as SARS and COVID-19 with therapeutic CS [8, 9]. Evidence showed that CS had a direct effect on increasing muscle protein degradation and inhibiting amino acid transport to muscle cells $[10,11]$. CS also induced electrolyte abnormalities, such as lower serum magnesium $(\mathrm{Mg})$ in patients [12]. $\mathrm{Mg}$ deficiency was associated with the increase of numerous clinical risks, including sarcopenia, vascular and metabolic disorders, insulin resistance, alterations in lipid metabolism [13], which were just similar to Cushing's symptoms. There was a vicious circle between $\mathrm{Mg}$ deficiency and metabolic diseases, while $\mathrm{Mg}$ supplementation benefited the patients in alleviating the symptoms [14, 15]. Evidence showed that $\mathrm{Mg}$ deficiency was associated with impaired muscle function [16]. The serum $\mathrm{Mg}$ concentration was independently correlated with muscle performance in the elderly [17], and Mg supplementation benefited muscle strength in humans [18]. Thus, besides direct effects, CS could also impair the skeletal muscle indirectly through CS-induced Mg deficiency.

We hypothesized that $\mathrm{Mg}^{++}$supplementation could alleviate CS-induced muscle atrophy. A rat model with CSassociated muscle atrophy symptom was used in this study [19]. The serum $\mathrm{Mg}^{++}$level, tissue composition, muscle function and morphology were evaluated to understand the effect of $\mathrm{Mg}^{++}$supplementation in vivo. The direct effects of CS and $\mathrm{Mg}^{++}$on myoblasts in vitro were also investigated. The in vivo mRNA expression of muscle atrophy marker genes MuRF1 and MAFbx were examined. The results of this study demonstrated for the first time that the CS-associated muscle atrophy was alleviated by $\mathrm{Mg}^{++}$ supplementation.

\section{Materials and methods}

\section{Ethics statement}

All the experimental protocols were reviewed and approved by the Animal Experimental Ethics Committee of the Chinese University of Hong Kong (Ref No. 17-087-MIS). We followed both the Guide for the Care and Use of Laboratory Animal (1996) [20] and the ARRIVE (Animals in research: reporting in vivo experiments) guidelines [21].

\section{Experimental animals and study design}

Eighteen 24-week-old male Sprague-Dawley rats with body weight of 500 550 g were used in this study. They were housed in a standard temperature-controlled animal room $\left(25{ }^{\circ} \mathrm{C}\right)$ and received food and water ad libitum.

Animals were randomly divided into three groups $(n=6)$. Twelve rats were used to induce CS-associated muscle atrophy with the induction procedure as follows: on day 1, under general anesthesia with i.p. injection of $90 \mathrm{mg} / \mathrm{kg}$ of ketamine and $10 \mathrm{mg} / \mathrm{kg}$ of xylazine, each rat was intravenously injected with $0.2 \mathrm{mg} / \mathrm{kg}$ of lipopolysaccharide (LPS, Escherichia coli O111:B4; Sigma-Aldrich, St. Louis, MO, USA) via tail vein with a controlled stable speed of injection steadily over $30 \mathrm{~min}$ for each rat to mimic the clinical inflammatory diseases to be indicated for CS therapy [19]. Clinically, high dose MPS (30 mg/kg) was prescribed to severe sepsis patients [22]. The dosage for rats was calculated based on a guide for dose conversion between animals and human [23]. Long-term CS therapy was used for the treatment of various clinical conditions [24]. To simulate long-term CS therapy on severe infection, on day 2, 3, 4 of the first week, three intraperitoneally injections of high-dose CS methylprednisolone (MPS, $100 \mathrm{mg} / \mathrm{kg}$ body weight, Pfizer Manufacturing Belgium NV) were given, and then low doses of MPS (40 mg/kg, i.p.) were given three times a week from the second week through week 6 . Week 6 was selected as the time point for efficacy assessment based on the previous dual-energy X-ray absorptiometry (DXA) results that shorter treatment could not induce lower muscle mass in rat [19]. Six rats without LPS + CS treatment were used as normal controls. Saline was used as the vehicle control with the same injection procedures as LPS and MPS treatments. The LPS + CS-treated rats 
were randomly divided into the $\mathrm{Mg}^{++}$supplementation group (LPS + CS + Mg group) and the LPS + CS group. For the LPS + CS + Mg group, from the first day of MPS injection, magnesium sulfate $\left(\mathrm{MgSO}_{4}\right)$ dissolved in drinking water (providing $50 \mathrm{mg} / \mathrm{kg} /$ day $\mathrm{Mg}^{++}$) was orally administrated daily to rats with oral gavage [25]. Drinking water was administrated daily to the normal group and LPS + CS group with oral gavage. The body weight of the rats was monitored twice a week. At the end of week 6 , the rats were euthanized by intravenous injection of pentobarbital, and samples were collected for further analysis (Fig. 1).

\section{Animal dual-energy X-ray absorptiometry (DXA)}

For determination of the tissue composition of lean weight and fat percent, at week 6 before euthanasia, rats were scanned and analyzed using UltraFocus ${ }^{\mathrm{TM}}$ Digital Radiography System (Faxitron Bioptics, LLC, USA) with $\times 1.0$ magnification at the lower body. Limited by the animal DXA machine, a whole-body scan could only be carried out on mice in our setting. The region of interest (ROI) was circled manually as an oval shape with a $0.8-1.0 \mathrm{~cm}^{2}$ size adjacent to the lateral tibia but not including any bone tissue. The ROI was chosen at the low limb excluded the bone region because the fat percent in normal rat was as low as $1.5 \%$ in this ROI to study the tissue composition alterations in the LPS + CS-treated groups [19].

\section{Ex vivo muscle functional test}

At week 6 before euthanasia and under general anesthesia, the extensor digitorum longus (EDL) of the left hind limb was dissected. CS was catabolic for fast-twitch muscles (e.g., EDL) while slow-twitch muscles (e.g., soleus) were more resistant to CS [26], the EDL was, therefore, chosen for analysis in the present study. For stabilization, the freshly isolated muscle was incubated in the Krebs solution with bubbled gas $\left(95 \% \mathrm{O}_{2}\right.$ and $\left.5 \% \mathrm{CO}_{2}\right)$ for $15 \mathrm{~min}$. After stabilization, the muscle function was investigated with the dualmode muscle lever arm system (305C-LR, Aurora Scientific Inc., Newmarket, Canada) [27, 28]. For the warm-up, two tetanic contractions (10 pulses with the Pulse Frequency $200 \mathrm{~Hz}$ and Pulse Width $0.2 \mathrm{~ms}$ ) with a 3-min rest interval were induced before the functional test. Optimal length $\left(L_{0}\right)$ was measured from a serial isometric twitch (one pulse with $0.2 \mathrm{~ms}$ width) with 30 -s intervals with increasing muscle length until maximal force. Under $L_{0}$, the Twitch force $\left(F_{0}\right)$ was the max force generated by a single stimulus (one pulse with $0.2 \mathrm{~ms}$ width). Under $L_{0}$, the Tetanic force $\left(F_{\mathrm{t}}\right)$ was the max force generated by tetanic contractions ( 80 pulses and the Pulse Frequency: $200 \mathrm{~Hz}$, Pulse Width $0.2 \mathrm{~ms}$ ). 30-s

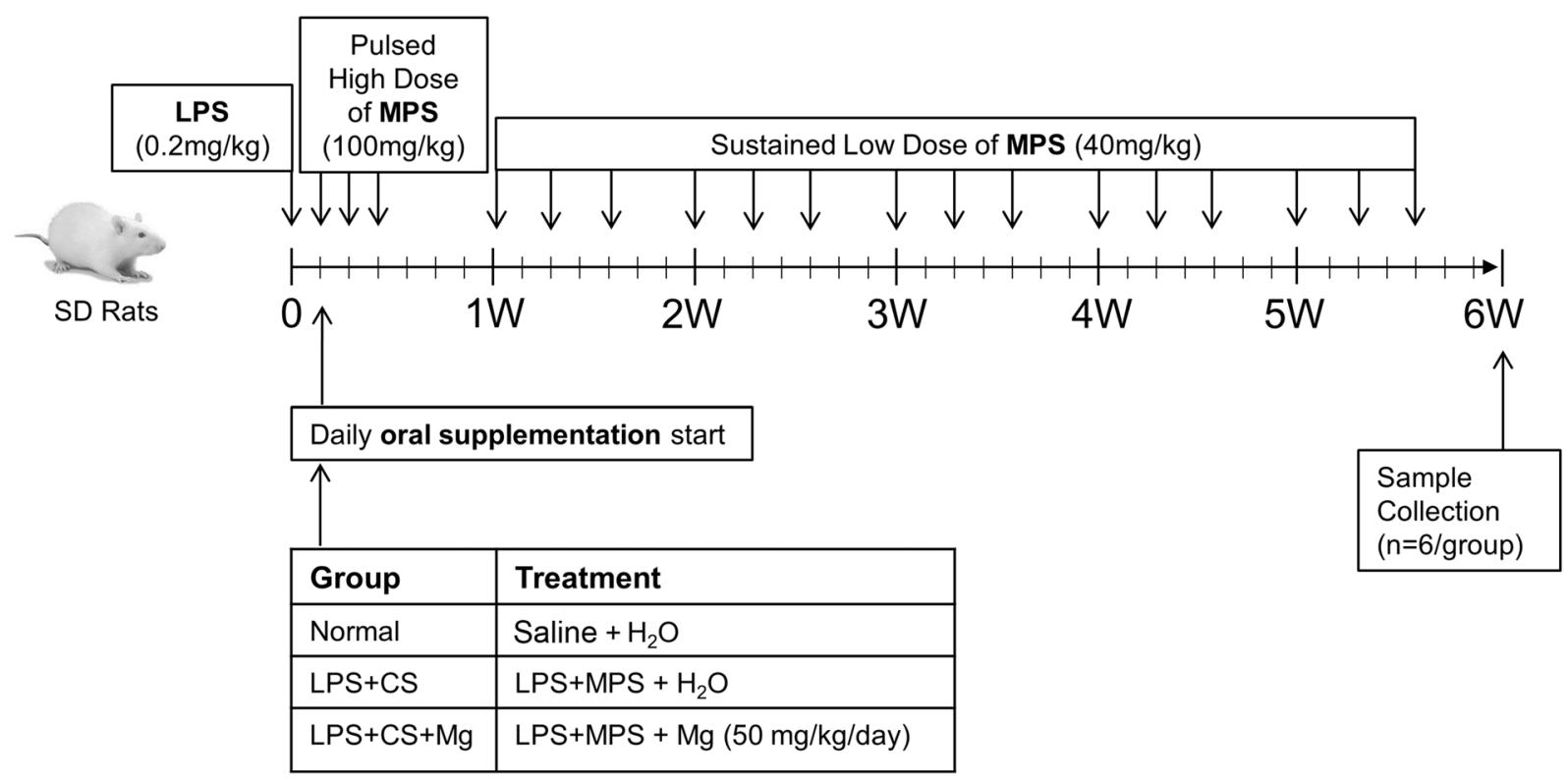

Fig. 1 Animal treatment protocols. Rats were treated with one injection of lipopolysaccharide (LPS) for simulating inflammation diseases and subsequent injections of CS methylprednisolone (MPS) for disease treatment for 6 weeks. Daily oral supplementation of $\mathrm{Mg}^{++}$ was performed from the first day of MPS injection for 6 weeks before sample collection 
rest was needed after each twitch stimulation, and 3-min rest was needed after each tetanic stimulation. The muscle mass was measured immediately after the functional test. The Dynamic Muscle Control system (DMC v5.4; Aurora Scientific, Inc.) was used to record muscle functional test results, and the Dynamic Muscle Analysis system (DMA v3.2; Aurora Scientific, Inc.) was used for the analysis of the functional test results.

The physiological cross-sectional area (PCSA) was calculated by dividing the muscle mass (MM) with the muscle optimal length $\left(L_{0}\right)$ and the density $(D)$ of mammalian skeletal muscle $\left(1.06 \mathrm{mg} / \mathrm{mm}^{3}\right)$. Normalized by PCSA, the specific twitch force $\left(\mathrm{SF}_{0}\right)$ and specific tetanic force $\left(\mathrm{SF}_{\mathrm{t}}\right)$ were obtained as the following equations [27]:

$$
\begin{aligned}
& \operatorname{PCSA}\left(\mathrm{mm}^{2}\right)=\frac{1000(\mathrm{mg} / \mathrm{g}) \times \mathrm{MM}(\mathrm{g})}{D\left(\mathrm{mg} / \mathrm{mm}^{3}\right) \times 10(\mathrm{~mm} / \mathrm{cm}) \times L_{0}(\mathrm{~cm})} \\
& \mathrm{SF}_{0}\left(\mathrm{mN} / \mathrm{mm}^{2}\right)=\frac{F_{0}(\mathrm{mN})}{\operatorname{PCSA}\left(\mathrm{mm}^{2}\right)} \\
& \mathrm{SF}_{\mathrm{t}}\left(\mathrm{mN} / \mathrm{mm}^{2}\right)=\frac{F_{0}(\mathrm{mN})}{\operatorname{PCSA}\left(\mathrm{mm}^{2}\right)}
\end{aligned}
$$

\section{Histological and immunofluorescence analyses}

At week 6 before euthanasia, the EDL of the right hind limb was dissected under general anesthesia, and then frozen immediately in liquid nitrogen-chilled 2-methylbutane in optimal length for $20 \mathrm{~s}$. The frozen muscles were stored at $-80{ }^{\circ} \mathrm{C}$, then transverse sectioned with $8 \mu \mathrm{m}$ thickness using a cryostat (Cryocut 1800 , Leica) at $-20^{\circ} \mathrm{C}$ and stored at $-80{ }^{\circ} \mathrm{C}$ for further analysis. After cryosection, the other half of EDL was stored at $-80{ }^{\circ} \mathrm{C}$ for mRNA quantification.

The cryosections were thawed at room temperature, fixed in $10 \%$ buffered formalin for $10 \mathrm{~min}$, and then stained with hematoxylin and eosin (H\&E) and Oil Red O for histological evaluations of muscle fiber and intramuscular fat infiltration. Briefly, for H\&E staining, the fixed cryosections were stained with hematoxylin for $1 \mathrm{~min}$, differentiated and blued and then stained with eosin for $2 \mathrm{~min}$. For Oil Red O staining, the fixed cryosections were stained with $0.5 \%$ Oil Red O working solution in isopropanol for $15 \mathrm{~min}$ and counterstained with hematoxylin for $30 \mathrm{~s}$ after washed with distilled water. Digital images were captured (Leica DM6000B, Leica Micro-systems, Wetzlar, Germany). The proportion of muscle fiber area to total area of the muscle cross-section and the muscle fiber cross-sectional area (CSA) were determined on $\mathrm{H} \& \mathrm{E}$-stained images with the aid of image morphometry software Image J (ImageJ 1.51j8, National Institutes of Health, USA). The outline of the individual fibers was traced, and the area was expressed in $\mu \mathrm{m}^{2}$. For analyzing intramuscular fat infiltration, the proportion of Oil Red O-stained area to total area was determined on Oil Red O-stained images with Image J (ImageJ 1.51j8, National Institutes of Health, USA). The area of red staining was automatically differentiated and calculated by the default program.

For muscle fiber typing, immunofluorescence (IF) staining of myosin heavy chain (MHC) was performed with a primary antibody cocktail including antibodies against MHC I (BA-F8), MHC IIa (SC-71), and MHC IIb (BF-F3) (DSHB, IA, USA), and the secondary antibody cocktail including Alexa Fluor $350 \mathrm{IgG} 2 \mathrm{~b}$, Alexa Fluor $488 \mathrm{IgG1}$, and Alexa Fluor 555 IgM (Invitrogen, CA, USA). The concentrations of both cocktails were $4 \mu \mathrm{g} / \mathrm{mL}[29]$. After the image acquisition with a microscopic imaging system (Leica DM6000B, Leica Microsystems, Wetzlar, Germany), areas of MHC I (blue), MHC IIa (green), MHC IIb (red) were quantified with Image J (ImageJ 1.51j8, National Institutes of Health, USA). Data were expressed with the area proportion and the CSA of the different fiber types.

\section{Serum $\mathbf{M g}^{++}$}

Serum was collected at week 6 and stored at $-80{ }^{\circ} \mathrm{C}$. Colourimetric test was performed to determine the serum $\mathrm{Mg}^{++}$ level using Mg LiquiColor ${ }^{\circledR}$ kit (Stanbio Laboratory, Texas, USA).

\section{Cell culture}

The mouse myoblast cell line C2C12 (ATCC, Rockville, $\mathrm{MD}$, USA) was grown in the growth medium, which was DMEM containing $10 \% \mathrm{FBS}$ in a $5 \% \mathrm{CO}_{2}$ humidified atmosphere at $37{ }^{\circ} \mathrm{C}$. For myoblast differentiation, the growth medium in $\mathrm{C} 2 \mathrm{C} 12$ myoblast cultures was replaced by the differentiation medium, which was DMEM containing $2 \%$ horse serum (HS) in a $5 \% \mathrm{CO}_{2}$ humidified atmosphere at $37{ }^{\circ} \mathrm{C}$. The medium was changed every day. After 2 days of incubation with differentiation medium, cells were treated with CS $(1 \mu \mathrm{M}$ MPS $)$ with or without $10 \mathrm{mM}$ magnesium chloride for 2 days. The control group was treated with solvent only. After 2 days of MPS or solvent treatment, the myotube diameter was measured and the total mRNA was extracted for quantification. 


\section{Measurement of myotube diameter}

Myotube cultures were photographed under a phasecontrast microscope at $\times 100$ magnification on day 4 . The myotube diameters were measured from 10 random fields using ImageJ software (ImageJ 1.51j8, National Institutes of Health, USA).

\section{Quantitative real-time PCR (qPCR) analysis}

MyoD, MyoG, Myf-5, Myf-6, Foxo3, MuRF1, and MAFbx mRNA levels were quantified by real-time PCR. GAPDH mRNA was used as the endogenous control for normalization. Total RNA was extracted using RNeasy Mini Kit (Qiagen, USA). Quantity and quality of RNA were measured using a Nanodrop (ND-2000, Thermo Scientific, Wilmington, DE, USA). The cDNA was synthesized using the PrimeScript RT Reagent Kit with gDNA Eraser (TaKaRa). The real-time PCR was performed using SYBR ${ }^{\circledR}$ Premix Ex Taq ${ }^{\mathrm{TM}}$ (Tli RNaseH Plus, TaKaRa) and further processed using a QuantStudio ${ }^{\mathrm{TM}} 12 \mathrm{~K}$ Flex Real-Time PCR system (Life Technologies, Thermo Fisher Scientific). The 2- $\Delta \Delta \mathrm{CT}$ method was used to calculate the relative mRNA expression. The sequences of the forward and reverse primers were listed as below: MyoD: 5'- CCA CTC CGG GAC ATA GAC TTG -3', 5'-AAA AGC GCA GGT CTG GTG AG -3'; MyoG: 5'- GAG ACA TCC CCC TAT TTC TAC CA -3', 5'- GCT CAG TCC GCT CAT AGC C -3'; Myf-5: 5'- CAC CAC CAA CCC TAA CCA GAG -3', 5'- AGG CTG TAA TAG TTC TCC ACC TG -3'; Myf-6: 5' - AGA GGG CTC TCC TTT GTA TCC -3', 5'- CTG CTT TCC GAC GAT CTG TGG -3'; Foxo3: 5' - CTG GGG GAA CCT GTC CTA TG -3', 5'- TCA TTC TGA ACG CGC ATG AAG -3'; MuRF1: 5'- CCA GGC TGC GAA TCC CTA C -3', 5'- ATT TTC TCG TCT TCG TGT TCC TT -3'; MAFbx: 5'- CAG CTT CGT GAG CGA CCT C -3', 5'GGC AGT CGA GAA GTC CAG TC -3'; and GAPDH: 5' - CAT GGC CTT CCG TGT TCC TA -3', 5' - CCT GCT TCA CCA CCT TCT TGA T -3 '.

\section{Statistical analysis}

Results were expressed as mean \pm SD with one-way ANOVA or two-way repeated measures ANOVA if the data were with different time points, followed by Bonferroni post-test to assess statistical significance. Statistical analysis was performed using SPSS 17.0 software (Chicago, IL, USA). $p<0.05$ was considered significant.

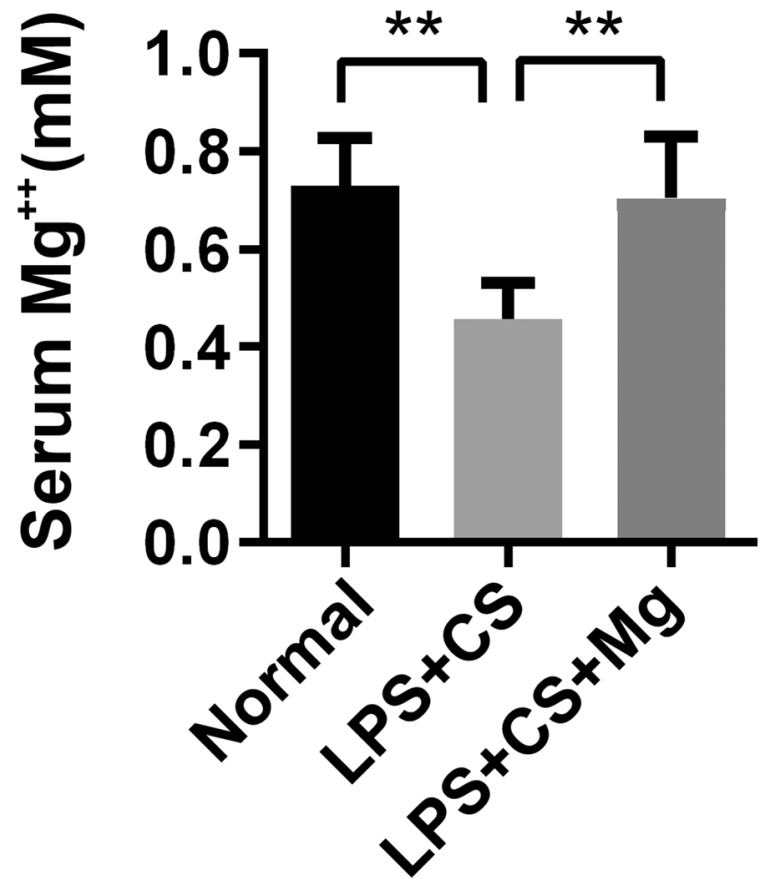

Fig. 2 Serum $\mathrm{Mg}^{++}$. Comparison of the serum $\mathrm{Mg}^{++}$level among the three groups at week 6 after LPS + CS treatment. $* * p<0.01$

\section{Results}

\section{$\mathrm{Mg}^{++}$supplement corrects serum $\mathrm{Mg}^{++}$level in LPS + CS-treated rats}

We tested the serum $\mathrm{Mg}^{++}$levels among the three groups at week 6 after LPS + CS treatment. Results showed that the LPS + CS group had $37.4 \%$ lower serum $\mathrm{Mg}^{++}$levels $(0.46 \pm 0.07 \mathrm{mM})$ than the normal group $(0.73 \pm 0.10 \mathrm{mM})$ $\left(p<0.01\right.$, Fig. 2). The serum $\mathrm{Mg}^{++}$levels of rats in the LPS + CS group were below the normal range in the current study $(0.53 \sim 0.93 \mathrm{mM}$, mean $\pm 2 \mathrm{SD})$. $\mathrm{Mg}^{++}$supplementation group had $54.3 \%$ higher serum $\mathrm{Mg}^{++}$levels $(0.71 \pm 0.13 \mathrm{mM})$ compared to that in the LPS + CS group $\left(p<0.01\right.$, Fig. 2). The serum $\mathrm{Mg}^{++}$levels of rats in the LPS $+\mathrm{CS}+\mathrm{Mg}$ group were within the normal physiological range.

\section{$\mathrm{Mg}^{++}$regulates tissue composition in LPS + CS-treated rats}

The LPS + CS group had $12.8 \%$ decreased body weight 3 days after the first LPS injection $(p<0.01$ compared with earlier time point, Fig. 3A), and after that, the body weight 


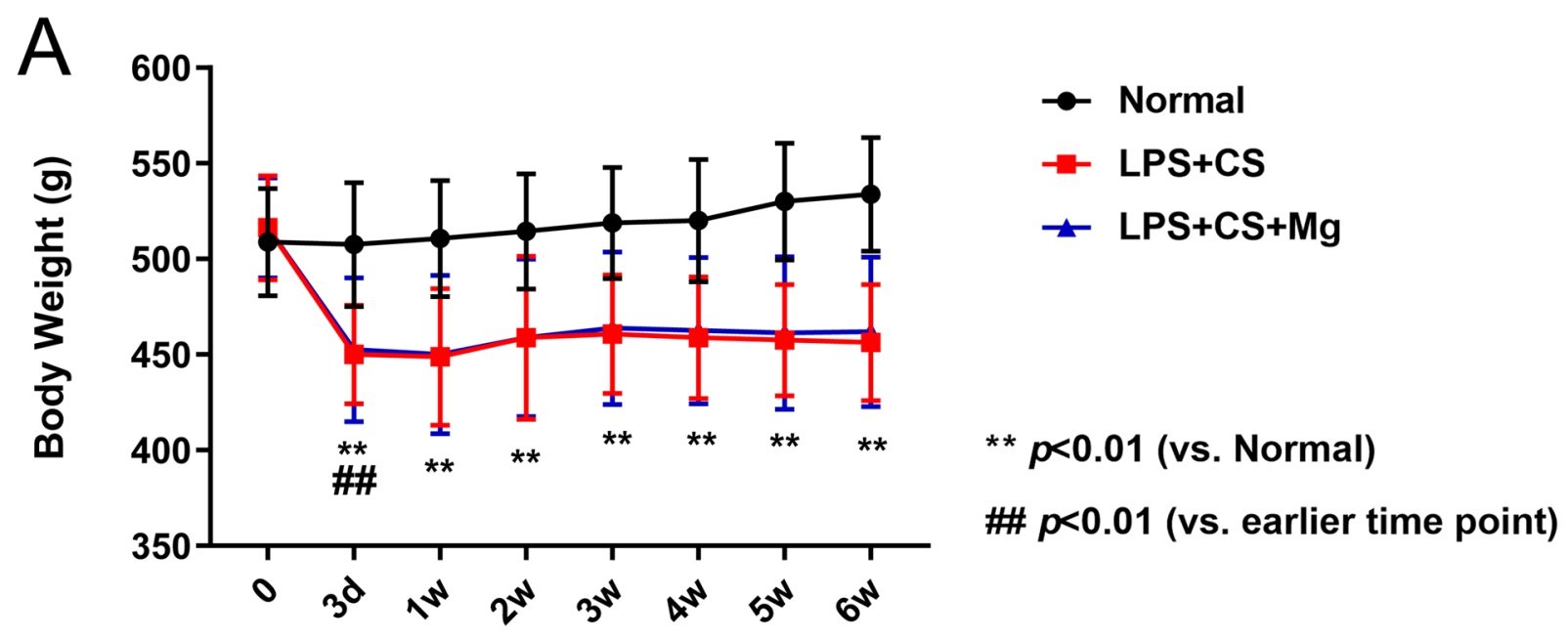

B

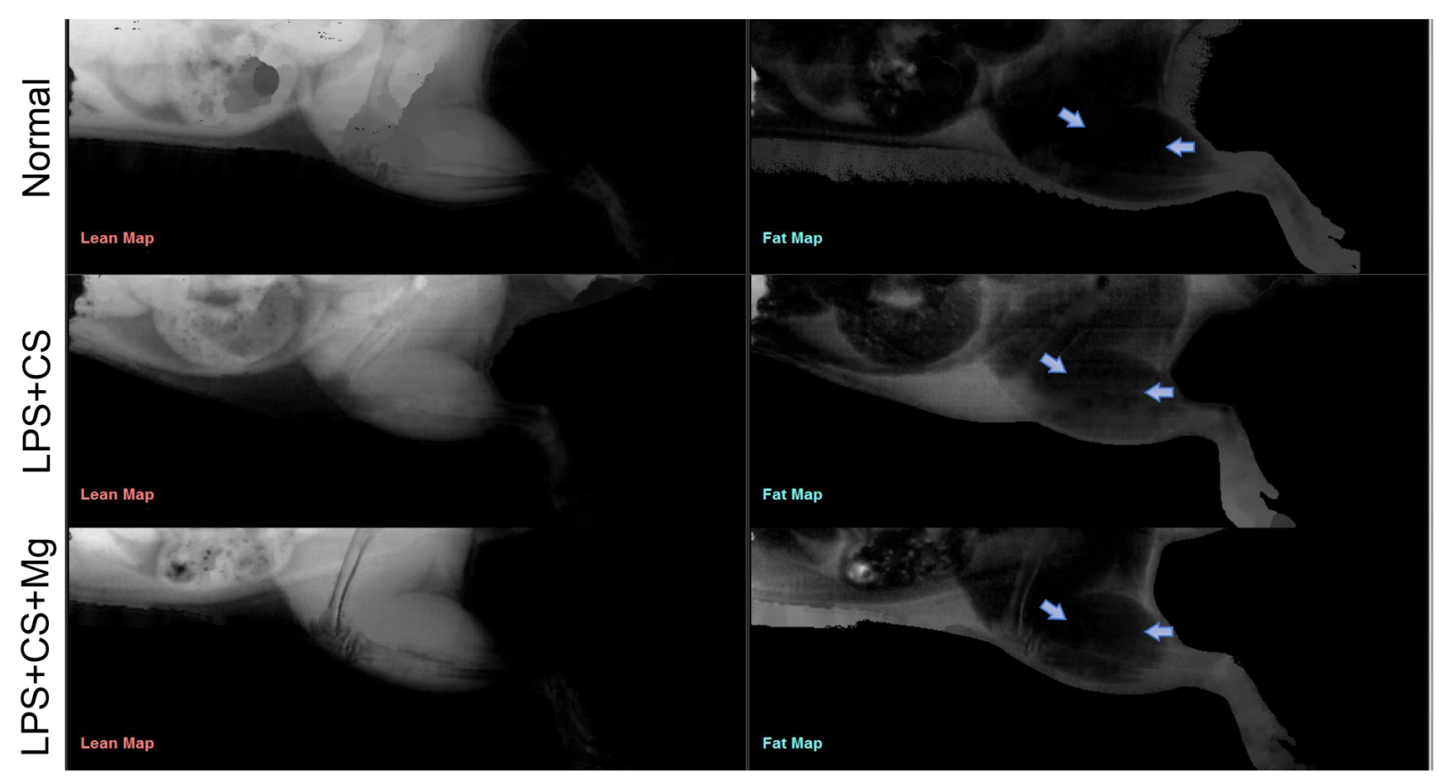

0

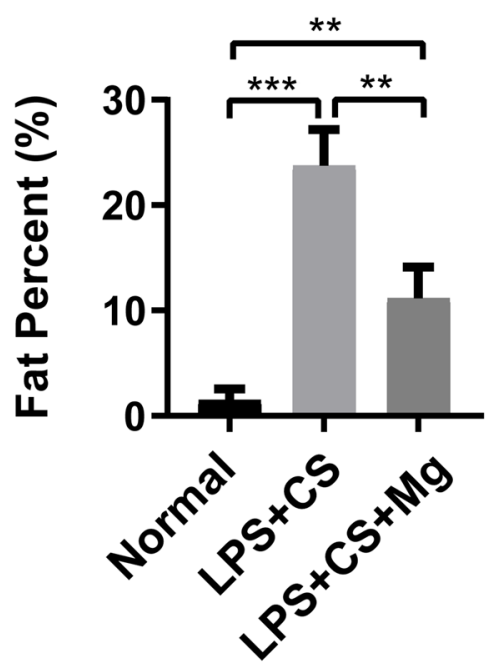

D

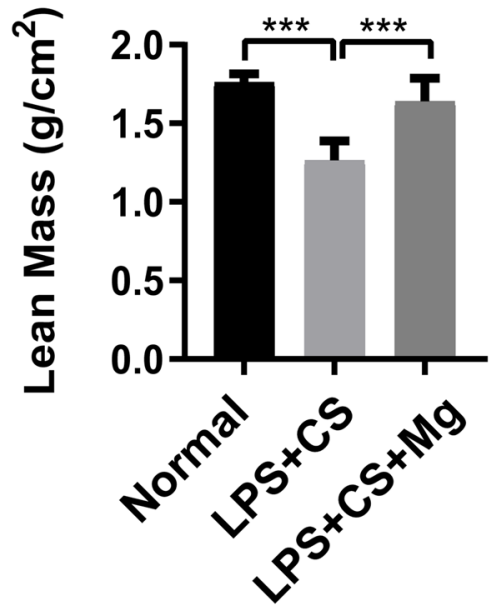


4Fig. $3 \mathrm{Mg}^{++}$regulates tissue composition in CS-treated rats. A The body weights of rats in $\mathrm{CS}$ group and $\mathrm{CS}+\mathrm{Mg}$ group were significantly decreased 3 days after the first LPS injection, and then consistently lower when compared to the normal rats. B Representative images showed animal dual-energy X-ray absorptiometry (DXA) scanning at the lower limb of rats after 6 weeks of treatment. The region of interest (ROI) was set at a region adjacent to the lateral tibia but not including any bone tissue (arrows). C Results showed that the fat percent of CS group was significantly higher, and the lean mass of CS group was significantly lower at lower limb when compared to normal group; but $\mathrm{Mg}^{++}$supplementation significantly decreased the fat percent and elevated the lean mass when compared to the CS group. $* * p<0.01, * * * p<0.001$

of the LPS + CS group was consistently lower than that of the normal group ( $p<0.01$, Fig. 3A). There was no difference between the LPS $+\mathrm{CS}+\mathrm{Mg}$ group and the LPS $+\mathrm{CS}$ group for the body weight ( $p>0.05$, Fig. 3A). Dual-energy $\mathrm{X}$-ray absorptiometry (DXA) analysis showed that after 6 weeks of treatment, the fat percentage of the CS group was 14.9-fold higher, and the lean mass of the CS group was $28.2 \%$ lower when compared to the normal group; $\mathrm{Mg}$ supplementation had $53.0 \%$ lower fat percent and $29.7 \%$ higher lean mass when compared to the CS group ( $p<0.01$ for all, Fig. 3B, C, D).

\section{$\mathrm{Mg}^{++}$preserves muscle function in LPS + CS-treated rats}

Ex vivo muscle function test was performed at week 6 on the extensor digitorum longus (EDL). The morphological parameters were measured during the ex vivo muscle function test. Results showed that the muscle mass of EDL in the LPS + CS group was $14.8 \%$ lower than that in the normal group ( $p<0.05$, Fig. $4 \mathrm{~A})$, and the optimal length $\left(L_{0}\right)$ in the LPS + CS group was $22.4 \%$ lower than that in the normal group ( $p<0.05$, Fig. 4B), while there was no significant difference between the LPS $+\mathrm{CS}+\mathrm{Mg}$ group and LPS $+\mathrm{CS}$ group or normal group ( $p>0.05$, Fig. $4 \mathrm{~A})$. There was no significant difference among groups for the physiological cross-sectional area (PCSA) $(p>0.05$, Fig. 4C). For the muscle strength, contractibility, and relaxation parameters under twitch stimulation (Fig. 4D), the specific twitch force in the LPS + CS group was $23.3 \%$ significantly lower than that in the normal group ( $p<0.01$, Fig. $4 \mathrm{E}$ ), while $\mathrm{Mg}^{++}$ treatment had $22.2 \%$ higher specific twitch force when compared to the LPS + CS group ( $p<0.05$, Fig. 4E). There was no significant difference for the time to max-force or the half-relaxation time among groups ( $p>0.05$, Fig. $4 \mathrm{~F}$, $\mathrm{G})$. Then we tested the muscle strength, contractibility, and relaxation parameters under tetanic stimulation (Fig. $4 \mathrm{H}$ ). The results showed that the specific tetanic force in the LPS + CS group was $40.3 \%$ lower than that in the normal group ( $p<0.001$, Fig. 4I), the time to max-force in the LPS + CS group was $17.9 \%$ shorter than that in the normal group ( $p<0.01$, Fig. $4 \mathrm{~J}$ ), whereas $\mathrm{Mg}^{++}$treatment group showed higher specific tetanic force $(42.0 \%)$ and longer time to max tetanic force $(18.9 \%)$ when compared to the LPS + CS group $(p<0.05$ for both, Fig. 4I, J). The halfrelaxation time for the LPS + CS group was $27.5 \%$ longer compared to that in the normal group ( $p<0.01$, Fig. $4 \mathrm{~K}$ ), but $\mathrm{Mg}^{++}$treatment group had $15.8 \%$ shorter half-relaxation time $(p<0.05$, Fig. $4 \mathrm{~K})$.

\section{$\mathrm{Mg}^{++}$protects muscle morphology in LPS + CS-treated rats}

Histology and histomorphometry analysis were performed on the transverse sectioned EDL 6 weeks after CS treatment. Quantification results on the H\&E-stained images showed that the proportion of muscle fiber area to total area of the LPS + CS group was $6.0 \%$ lower than normal $\left(p<0.01\right.$, Fig. 5A, D), and that $\mathrm{Mg}^{++}$supplementation group had $4.1 \%$ greater the proportion of muscle fiber area to total area when compared to the LPS + CS group $(p<0.05$, Fig. 5A, D). There was no significant difference in muscle fiber CSA among groups ( $p>0.05$, Fig. 5E). Oil Red O staining showed that LPS + CS treatment for 6 weeks induced fat infiltration between muscle fibers (Fig. 5B). Quantification of Oil Red O-stained images showed that the proportion of stained areas in LPS + CS group was 12.8fold higher than normal $(p<0.001$, Fig. $5 \mathrm{~F})$, but $\mathrm{Mg}^{++}$supplementation group had $63.6 \%$ lower the proportion of the stained area when compared to LPS + CS group $(p<0.001$, Fig. 5F). Myosin heavy chain (MHC) immunofluorescence staining results showed that the percentage area of type IIb in the LPS + CS group was $35.6 \%$ lower than normal $(p<0.01$, Fig. $5 \mathrm{C}, \mathrm{G})$, but that of type IIa was $71.9 \%$ higher than normal $(p<0.05$, Fig. $5 \mathrm{C}, \mathrm{G})$. LPS $+\mathrm{CS}+\mathrm{Mg}$ group showed $33.0 \%$ higher percentage of type IIb fibers than the LPS + CS group $(p<0.01$, Fig. 5C, G). The type IIb muscle fiber CSA of the LPS + CS group was $28.9 \%$ lower than normal $(p<0.01$, Fig. 5C, H). LPS $+\mathrm{CS}+\mathrm{Mg}$ group showed $29.4 \%$ higher type IIb muscle fiber CSA than the LPS + CS group ( $p<0.05$, Fig. 5C, H). No significant differences were detected for the type I or type IIa muscle fiber CSA among groups $(p>0.05$, Fig. $5 \mathrm{C}, \mathrm{H})$.

\section{$\mathrm{Mg}^{++}$inhibits $\mathrm{C} 2 \mathrm{C} 12$ myotube atrophy induced by CS in vitro}

We investigated the $\mathrm{Mg}^{++}$effect on myotube atrophy induced by $\mathrm{CS}$ using the $\mathrm{C} 2 \mathrm{C} 12$ myoblast cell line (Fig. 6A). The differentiation of C2C12-myoblast to myotube was observed 
Fig. $4 \mathrm{Mg}^{++}$preserves muscle function after CS treatment.

A-C Muscle parameters

measured during ex vivo muscle function test: A muscle mass, B optimal length $\left(L_{0}\right)$, and $\mathbf{C}$ physiological cross-sectional area (PCSA). D Representative force-time graphs during twitch stimulations. E-G Comparison of muscle strength, contractibility, and relaxation parameters under twitch stimulation: $\mathbf{E}$ specific twitch force, $\mathbf{F}$ time to max-force, and $\mathbf{G}$ half-relaxation time among groups. H Representative force-time graphs during tetanic stimulations. I-K Comparison of muscle strength, contractibility, and relaxation parameters under tetanic stimulation: I specific tetanic force, $\mathbf{J}$ time to max-force, and $\mathbf{K}$ half-relaxation time among groups. ${ }^{*} p<0.05, * * p<0.01$, $* * * p<0.001$
A
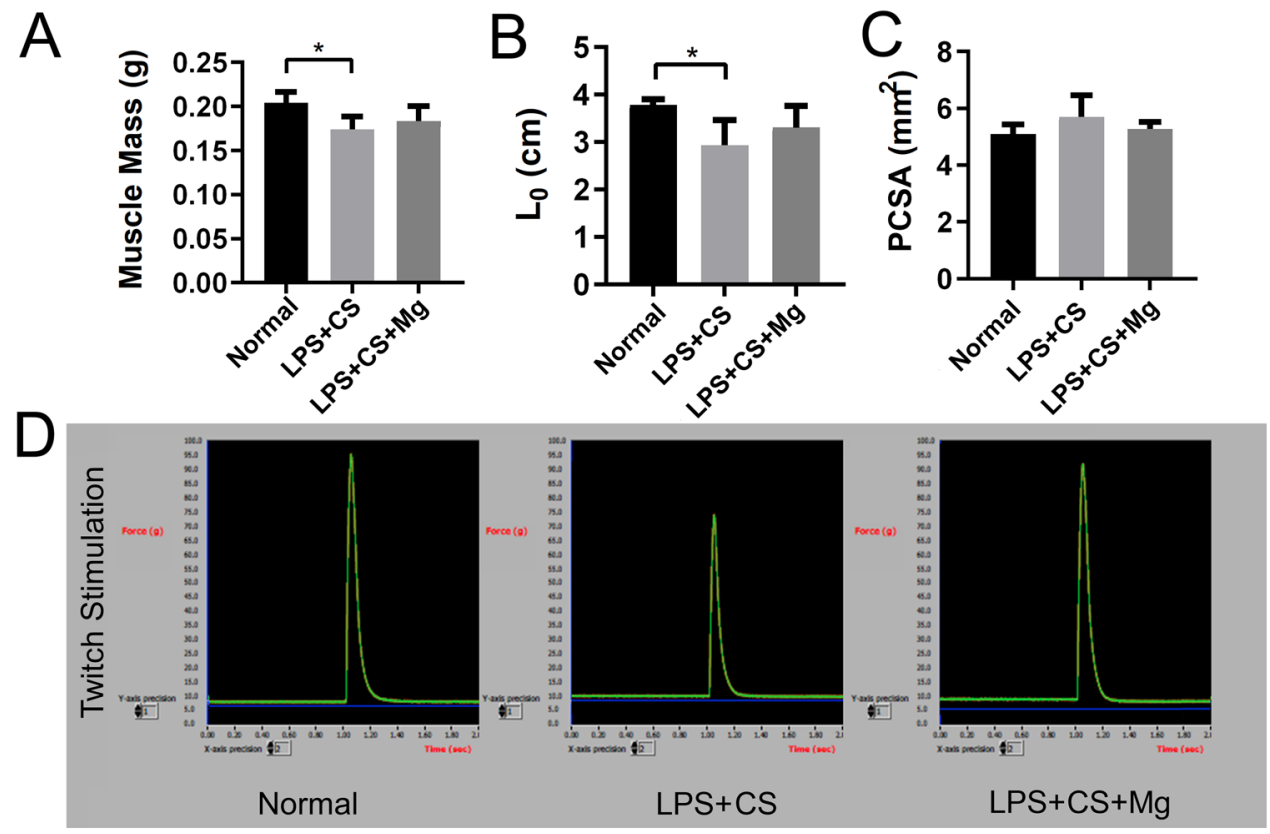

E
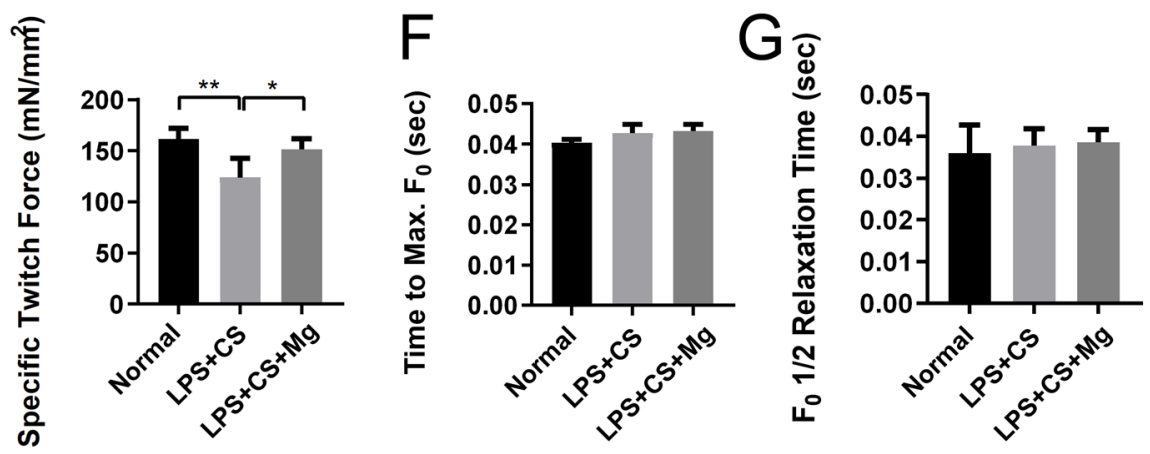

$\mathrm{H}$
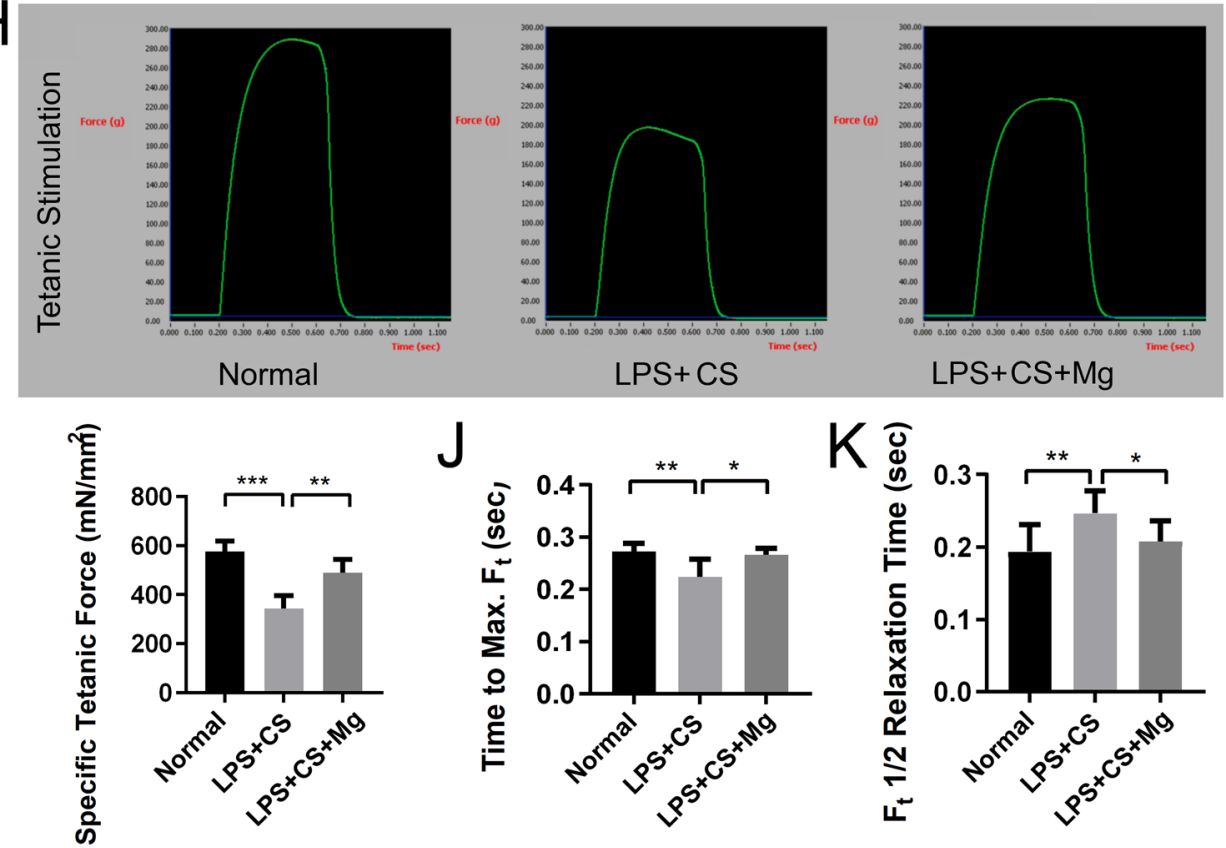
in phase-contrast brightfield images. Been treated with $2 \%$ HS for 4 days, the fusion of cells and the formation of elongated myotubes were observed (Fig. 6B). The qPCR analysis detected the expression of myogenic and atrophic markers in cultured myoblast after 4 days of their differentiation. Results showed that after 2 days of CS treatment during the differentiation from myoblast to myotube, the diameter of the $\mathrm{C} 2 \mathrm{C} 12$ myotubes was smaller than that of control group $(p<0.01$, Fig. $6 \mathrm{C}) . \mathrm{Mg}^{++}$treatment group had significantly larger diameter of the $\mathrm{C} 2 \mathrm{C} 12$ myotubes when compared to the CS group ( $p<0.05$, Fig. 6C). For the myoblast differentiation marker genes MyoD, MyoG, Myf-5, and Myf-6, there was no significant difference in their mRNA expression among groups $(p>0.05$, Fig. $6 \mathrm{D}-\mathrm{G})$. For the genes regulating protein degradation, the Foxo 3 mRNA expression was not significantly affected by $\mathrm{CS}$ or $\mathrm{Mg}^{++}$treatment ( $p>0.05$, Fig. $6 \mathrm{H}$ ), while the MuRF1 (official name Trim63) and MAFbx (official gene name Fbxo32) mRNA expressions were both significantly greater in CS treatment group $\left(p<0.01\right.$ for both, Fig. 6I, J). $\mathrm{Mg}^{++}$treatment group had significantly lower expressions of the MuRF1 and MAFbx mRNA compared to the CS group $(p<0.01$ for both, Fig. 6I, J).

\section{$\mathrm{Mg}^{++}$inhibits LPS + CS-induced gene expression associated with muscle atrophy in vivo}

We quantified the mRNA levels of MuRF1 and MAFbx in EDL in vivo. Results showed that LPS + CS group had $41.3 \%$ greater MuRF1 ( $p<0.01$, Fig. 7A) and 32.1\% greater MAFbx $(p<0.05$, Fig. 7B) mRNA expressions in EDL of rats. $\mathrm{Mg}^{++}$supplementation group had 39.6\% lower MuRF1 and $46.7 \%$ lower MAFbx mRNA expressions when compared to the CS group ( $p<0.01$ for both, Fig. 7A, B)

\section{Discussion}

The present study demonstrated that supplementation of $\mathrm{Mg}^{++}$alleviated CS-associated muscle atrophy in rats, primarily evidenced by the muscle function tests where LPS + CS-induced lower specific twitch force and the specific tetanic force, while $\mathrm{Mg}^{++}$supplementation resulted in greater specific twitch force and the specific tetanic force than those LPS + CS-treated animals. This might suggest that $\mathrm{Mg}^{++}$supplementation could become a cost-effective prevention strategy for CS-associated muscle atrophy. In fact, as CS-associated musculoskeletal disorders have significant implications in our clinics, recent efforts have been made to develop relevant prevention and treatments, including anti-adipogenic and muscle tonifying herbs for the prevention of CS-associated osteonecrosis [30-32].

$\mathrm{Mg}^{++}$is the fourth abundant cation in the body and is the second abundant within the cell. $\mathrm{Mg}^{++}$is a cofactor for more than 300 cellular enzymes involving in energy production and protein and nucleic acid synthesis [33]. CS therapy was reported to be associated with hypomagnesemia, i.e., if the serum $\mathrm{Mg}^{++}$concentration was below the normal range [34]. The causes of $\mathrm{Mg}^{++}$ deficiency in CS-treated patients were multi-factorial, including the primary disease itself, decreased $\mathrm{Mg}^{++}$ absorption, and increased urinary excretion of $\mathrm{Mg}^{++}[12$, 34]. Our data suggested that CS treatment induced $\mathrm{Mg}$ deficiency in the current rat experimental model, and that $\mathrm{Mg}^{++}$supplementation maintained or increased the serum $\mathrm{Mg}^{++}$level back to normal range. The study, therefore, revealed the beneficial effect of simple $\mathrm{Mg}^{++}$supplementation in the prevention of metabolic disorder associated with CS therapy.

CS-induced muscle atrophy in this rat model, as evidenced by the lower muscle mass measured by DXA, decreased weighting of EDL and lower optimal length of EDL confirmed by muscle functional test. The muscle functional results consistently demonstrated the CSinduced muscle weakness including a reduction in the specific twitch force and the specific tetanic force, which were consistent with previous studies and clinical situations [35-37]. In the LPS + CS group, the time to peak tetanic force was shorter and the half relaxation time was longer compared to those in the normal control group, also indicating that muscle weakness was induced by LPS + CS treatment. $\mathrm{Mg}^{++}$supplementation elevated muscle mass shown by DXA measurements, improved strength muscle function in the specific twitch force and the specific tetanic force, held the time to peak tetanic force and the half relaxation time, suggesting the beneficial effect of $\mathrm{Mg}^{++}$supplementation on attenuating CS-associated muscle atrophy and weakness.

For the relationship between $\mathrm{Mg}^{++}$and lipid metabolism, our histological results showed that $\mathrm{Mg}^{++}$supplementation significantly lowered the intramuscular fat infiltration induced by LPS + CS treatment, and DXA results showed that $\mathrm{Mg}^{++}$supplementation significantly lowered the fat percent in the lower body. Correspondingly, the previous study showed that $\mathrm{Mg}^{++}$supplementation significantly decreased the bone marrow fat area fraction in the same CS-treated rat model [25]. In vitro study suggested that $\mathrm{Mg}^{++}$did not affect pre-adipocyte differentiation [38], which suggested that $\mathrm{Mg}^{++}$indirectly decreased the fat 


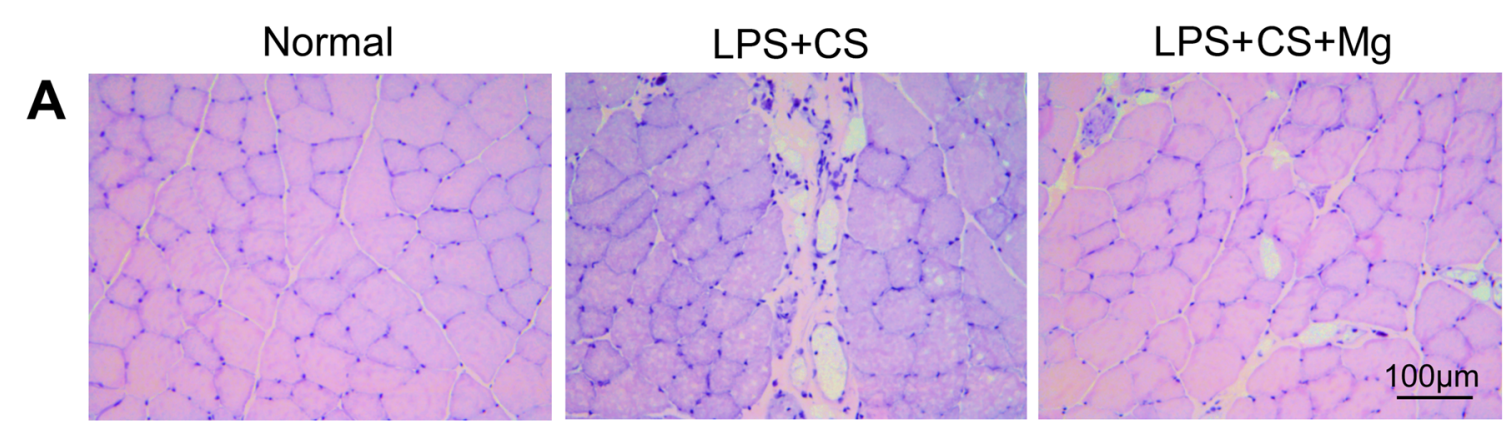

B
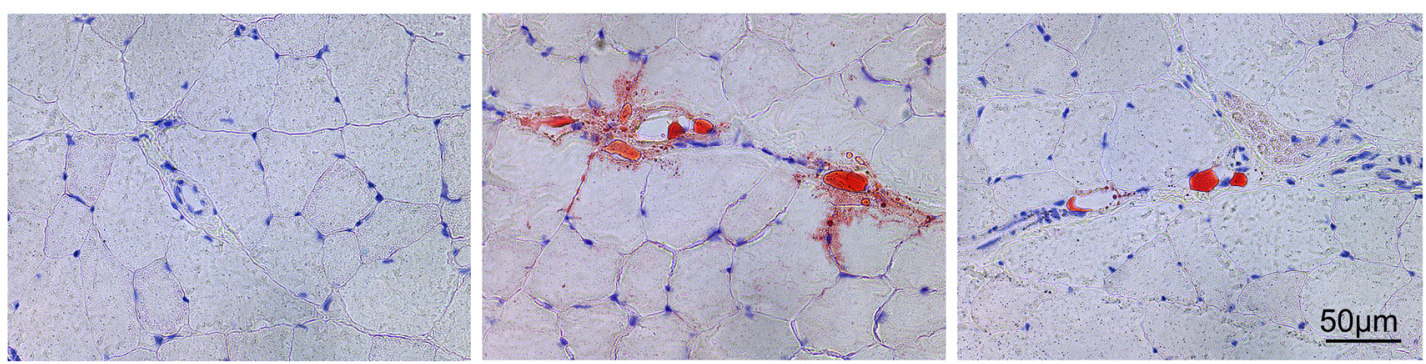

C
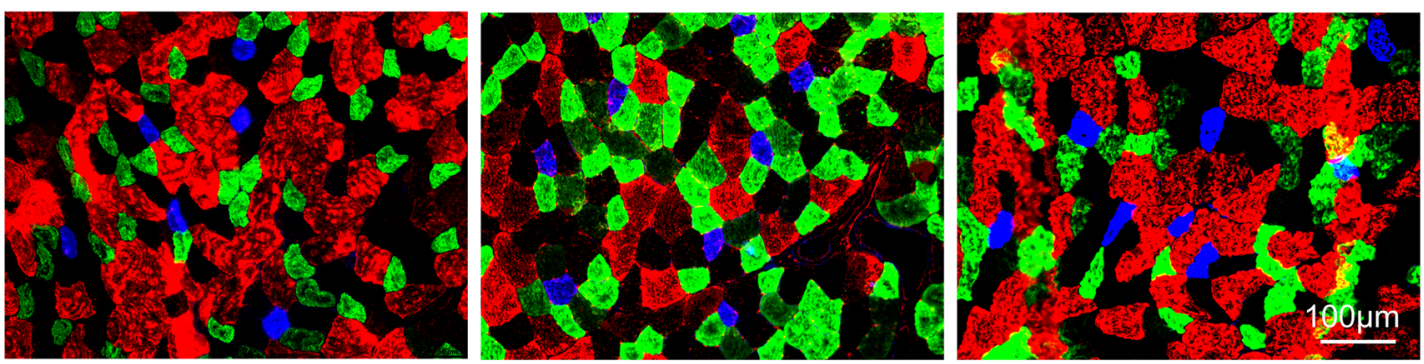

D

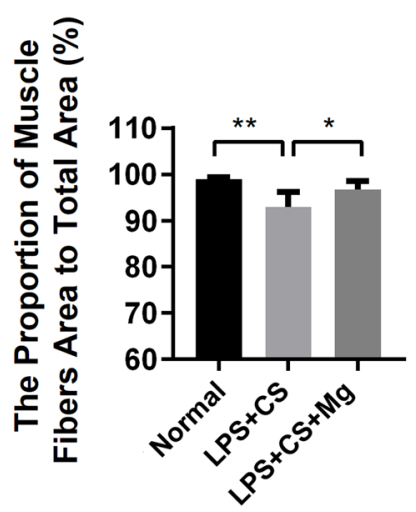

G

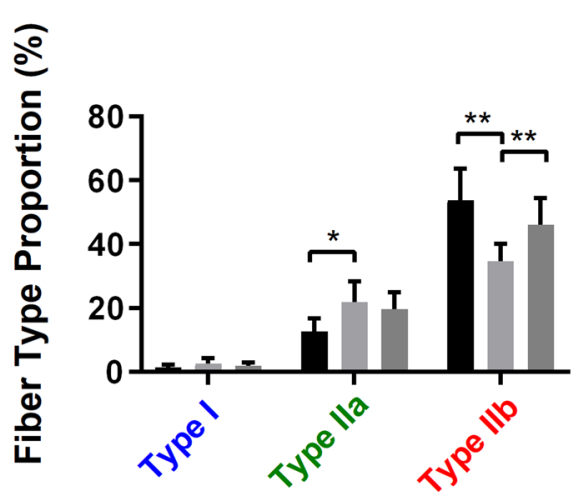

E

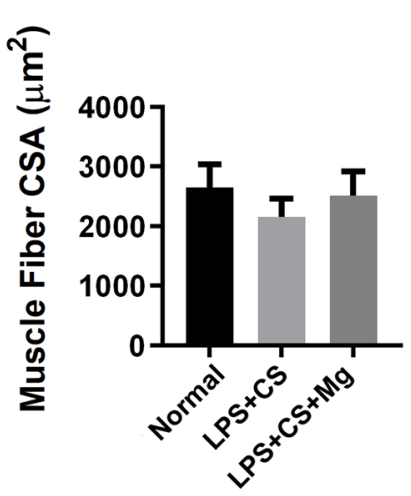

H
F

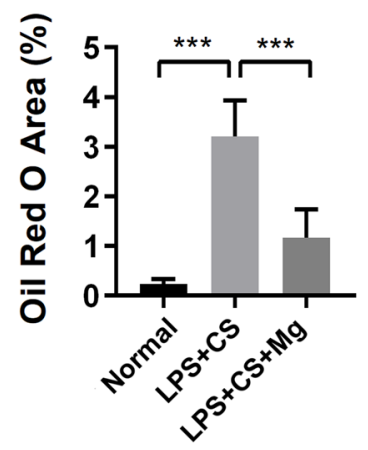

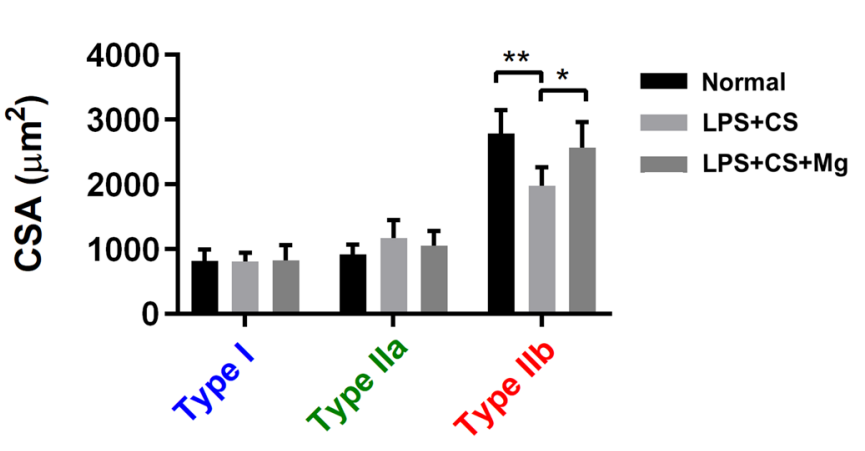


4Fig. $5 \mathrm{Mg}^{++}$protects muscle morphology. A Representative $\mathrm{H} \& \mathrm{E}$ staining of transverse sectioned EDL showed that LPS + CS treatment for 6 weeks induced fibrous and fatty tissue infiltration between muscle fibers. B Representative Oil Red O staining of transverse sectioned EDL showed that LPS + CS treatment for 6 weeks induced fat infiltrated between muscle fibers. C Representative myosin heavy chain (MHC) immunofluorescence (IF) staining of transverse sectioned EDL at week 6 after LPS + CS treatment. D Quantification results from $\mathrm{H} \& \mathrm{E}$-stained images showed the proportion of muscle fibers area to total area and $\mathbf{E}$ muscle fiber cross-sectional area (CSA). F Quantification results from Oil Red O-stained images showed the Oil Red O-stained area proportion. G Quantification results from MHC IF images showed the area proportion and $\mathbf{H}$ the CSA of fiber type I (blue), type IIa (green) and IIb (red). ${ }^{*} p<0.05$, $* * p<0.01, * * * p<0.001$

accumulation in muscle and bone marrow by regulating systemic lipid metabolism. Clinical studies showed that $\mathrm{Mg}^{++}$intake was negatively associated with the incidence of metabolic syndrome, i.e., there were inverse relations between $\mathrm{Mg}^{++}$intake and fasting glucose level, waist circumference, and blood triglycerides $[39,40] . \mathrm{Mg}^{++}$could modulate blood lipids as dietary $\mathrm{Mg}^{++}$contributed to the reduction of total serum cholesterol, low-density lipoprotein (LDL) cholesterol, and triglyceride [41]. It was demonstrated that serum lipid level was positively associated with muscle adiposity [42] and bone marrow fat [43]. Thus, the role of $\mathrm{Mg}^{++}$supplementation in intramuscular fat accumulation could be secondary to the effect on systemic lipid metabolism.

For the muscle fiber typing, the results of the current study demonstrated that CS treatment resulted in lower proportion of type IIb fiber area to total area and lower type IIb CSA. Although CS was reported to had pathologic changes to all types of muscle fibers [44], the previous study also demonstrated that the slow-twitch muscles were more resistant to CS [26], which supported the relevant finding of the current study. Similarly, the decline in muscle mass in the elderly was mainly due to the reduction in type II but not type I muscle fiber CSA
[45]. However, the LPS + CS group had higher proportion of type IIa fiber area to total area yet with no difference on type IIa CSA. The higher proportion area of type IIa might be compensatory to the lower proportion area of type IIb. $\mathrm{Mg}^{++}$supplementation significantly elevated the proportion of type IIb fiber area to total area and type IIb CSA when compared to the LPS + CS group, suggesting its protecting effect on muscle fiber IIb atrophy associated with CS treatment.

Muscle RING finger 1 (MuRF1) and muscle atrophy F-box (MAFbx) are markers of muscle atrophy. Report showed that they were muscle-specific E3 ubiquitin ligase that transcriptionally increased under muscle atrophy conditions [46]. Our in vitro study showed that CS group had lower diameter of the myotubes and greater MuRF1 and MAFbx expression, evidence that CS induced muscle atrophy by directly inducing muscle protein degradation. For the degradation of intact myofibrillar proteins, they were disassembled and removed from the myofibril by the calcium-dependent calpain system first and then degraded by the proteasome [47]. $\mathrm{Mg}^{++}$could act as a calcium antagonist to block the calcium-activated protein degradation [48]. The in vitro study indicated that $\mathrm{Mg}^{++}$supplementation reversed the CS-induced lower diameter of the myotubes and activated MuRF1 and MAFbx expression, thus providing evidence that $\mathrm{Mg}^{++}$protected muscle from atrophy by inhibiting muscle protein degradation. The in vivo MuRF1 and MAFbx expression were corresponding with the in vitro study.

In conclusion, $\mathrm{Mg}^{++}$supplementation promoted the anabolic effect on muscle function and morphology by inhibiting fat accumulation and muscle fiber protein degradation. The present study demonstrated for the first time that $\mathrm{Mg}^{++}$supplementation attenuated the adverse effect of CS treatment on muscle atrophy in a rat model. Our preclinical study provided a scientific foundation for facilitating the supplementation of $\mathrm{Mg}^{++}$as a potential clinical application for patients indicated for CS therapy. 


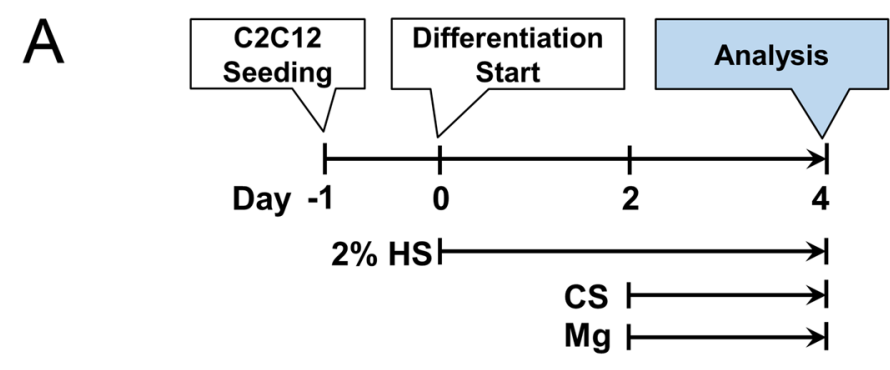

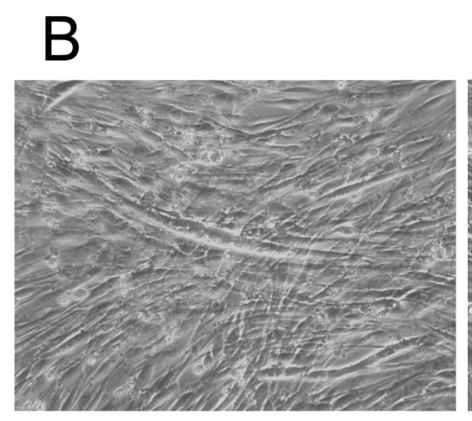

Control

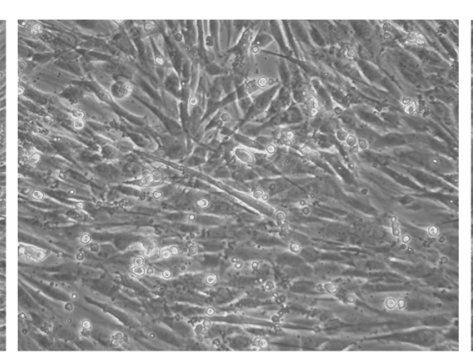

CS

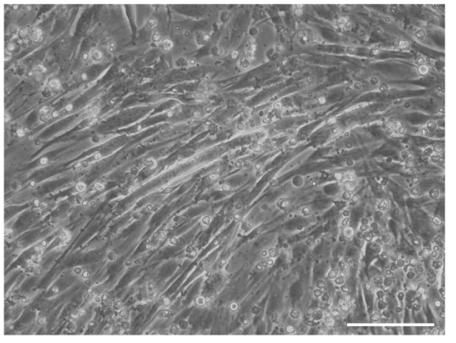

$\mathrm{CS}+\mathrm{Mg}$
C

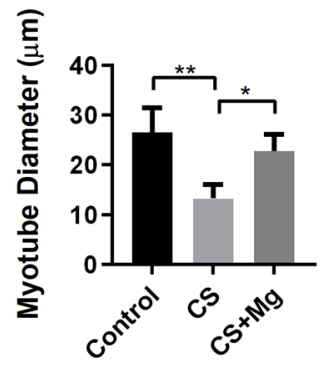

D

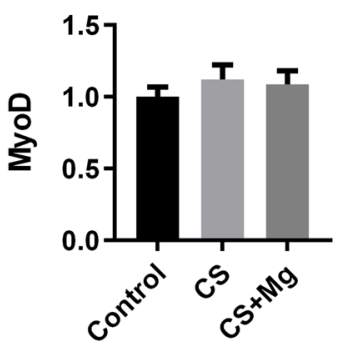

$\mathrm{H}$

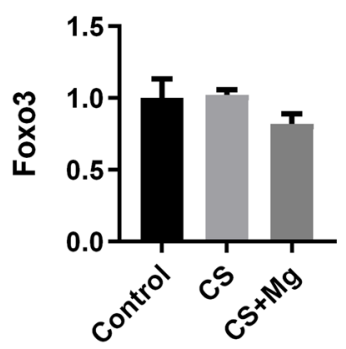

E
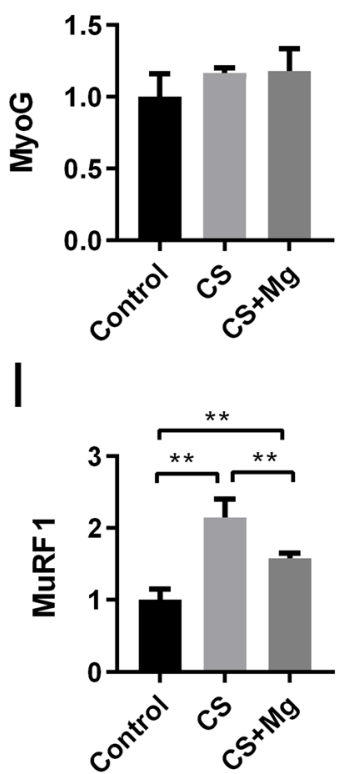

F

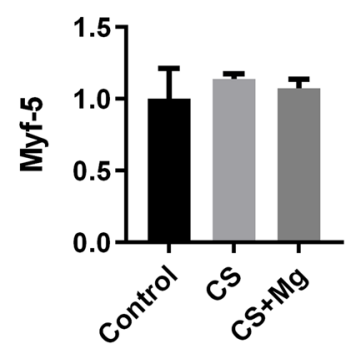

$J$

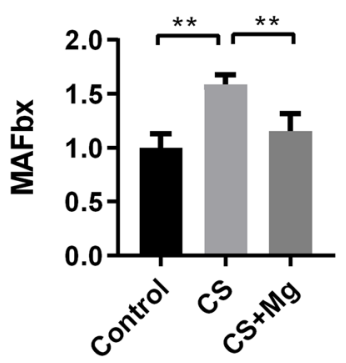

G

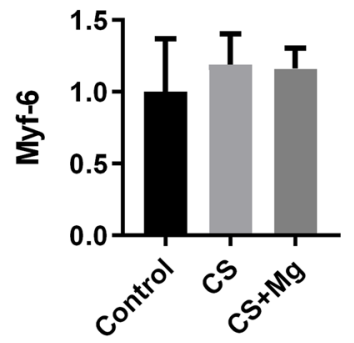

Fig. $6 \mathrm{Mg}^{++}$inhibits $\mathrm{C} 2 \mathrm{C} 12$ myotube atrophy induced by CS. A Procedure of the $\mathrm{C} 2 \mathrm{C} 12$ myotube atrophy experiment. B Representative images of myotubes after 4 days differentiation, scale bar $=50 \mu \mathrm{m}$. C The myotube diameter decreased 2 days after CS treatment. $\mathrm{Mg}^{++}$ significantly increased the myotube diameter when compared to the
CS group. D-J qPCR results showed the relative level of myogenic marker genes MyoD (D), MyoG (E), Myf-5 (F), and Myf-6 (G); and protein degradation regulating genes Foxo3 $(\mathbf{H})$, MuRF1 (I), and $\operatorname{MAFbx}(\mathbf{J})$ mRNA expressions among groups. ${ }^{* *} p<0.01, n=4$ 
Fig. $7 \mathrm{Mg}^{++}$inhibits muscle atrophy-associated gene expressions induced by CS in vivo. The qPCR results showed the relative level of muscle atrophy marker gene A MuRF1 and B MAFbx expressions in EDL 6 weeks after LPS + CS treatment. $* p<0.05, * * p<0.01$
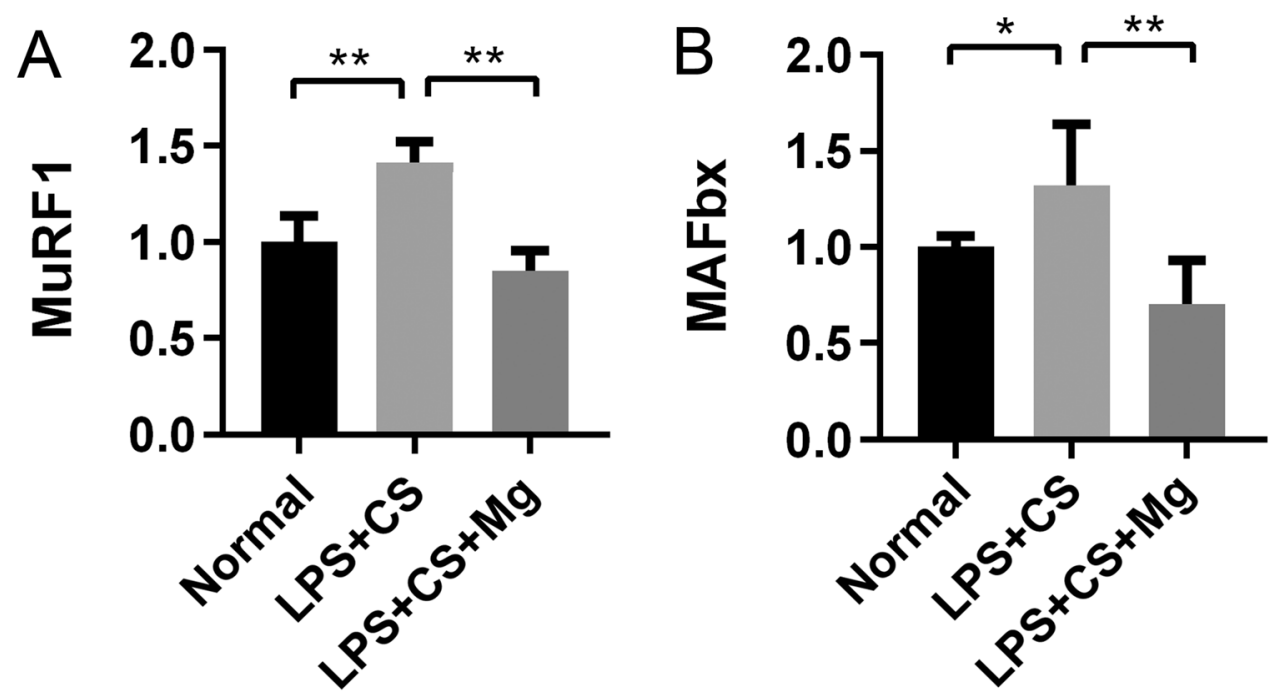

Acknowledgements The study was supported by the Theme-based Research Scheme of Research Grants Council of the Hong Kong Special Administrative Region, PR China (Ref No. T13-402/17-N), and the Research Funds from Health@InnoHK Program launched by Innovation Technology Commission of the Hong Kong SAR, P. R. China.

Author contributions LZ and LQ conceived and designed the study and wrote the original draft. LZ, LH, ZC, CC and RZ performed the experiments and analyzed data. LQ accepted responsibility for the integrity of the data analysis. All revised and edited the manuscript.

\section{Declarations}

Conflict of interest On behalf of all authors, the corresponding author states that there is no conflict of interest.

\section{References}

1. Lv H, de Vlas SJ, Liu W, Wang TB, Cao ZY, Li CP, Cao WC, Richardus JH (2009) Avascular osteonecrosis after treatment of SARS: a 3-year longitudinal study. Trop Med Int Health 14(Suppl 1):79-84

2. Ettinger WH, Goldberg AP, Applebaum-Bowden D, Hazzard WR (1987) Dyslipoproteinemia in systemic lupus erythematosus: effect of corticosteroids. Am J Med 83(3):503-508

3. Ledford $\mathrm{H}$ (2020) Coronavirus breakthrough: dexamethasone is first drug shown to save lives. Nature 582(7813):469-469

4. Buchman AL (2001) Side effects of corticosteroid therapy. J Clin Gastroenterol 33(4):289-294

5. Zhao D, Zhang F, Wang B, Liu B, Li L, Kim S-Y, Goodman SB, Hernigou P, Cui Q, Lineaweaver WC (2020) Guidelines for clinical diagnosis and treatment of osteonecrosis of the femoral head in adults (2019 version). J Orthop Transl 21:100-110

6. Cushing H (1964) The basophil adenomas of the pituitary body and their clinical manifestations (pituitary basophilism). J Neurosurg 21(4):318-347

7. Batchelor T, Taylor L, Thaler H, Posner J, DeAngelis L (1997) Steroid myopathy in cancer patients. Neurology 48(5):1234-1238
8. Webster JM, Fenton CG, Langen R, Hardy RS (2019) Exploring the interface between inflammatory and therapeutic glucocorticoid induced bone and muscle loss. Int J Mol Sci 20(22):5768

9. Disser NP, De Micheli AJ, Schonk MM, Konnaris MA, Piacentini AN, Edon DL, Toresdahl BG, Rodeo SA, Casey EK, Mendias CL (2020) Musculoskeletal consequences of COVID-19. JBJS 102(14):1197-1204

10. Löfberg E, Gutierrez A, Wernerman J, Anderstam B, Mitch W, Price S, Bergström J, Alvestrand A (2002) Effects of high doses of glucocorticoids on free amino acids, ribosomes and protein turnover in human muscle. Eur J Clin Invest 32(5):345-353

11. Pereira RMR, de Carvalho JF (2011) Glucocorticoid-induced myopathy. Jt Bone Spine 78(1):41-44

12. Das SK, Haldar AK, Ghosh I, Saha SK, Das A, Biswas S (2010) Serum magnesium and stable asthma: Is there a link? Lung India Off Organ Indian Chest Soc 27(4):205

13. Durlach J, Bac P, Durlach V, Rayssiguier Y, Bara M, Guiet-Bara A (1998) Magnesium status and ageing: an update. Magnes Res 11(1):25

14. Gommers LM, Hoenderop JG, Bindels RJ, de Baaij JH (2016) Hypomagnesemia in type 2 diabetes: a vicious circle? Diabetes 65(1):3-13

15. Durlach J, Durlach V, Bac P, Rayssiguier Y, Bara M, Guiet-Bara A (1993) Magnesium and ageing. II. Clinical data: aetiological mechanisms and pathophysiological consequences of magnesium deficit in the elderly. Magn Res 6(4):379-394

16. Rock E, Astier C, Lab C, Vignon X, Gueux E, Motta C, Rayssiguier Y (1995) Dietary magnesium deficiency in rats enhances free radical production in skeletal muscle. J Nutr 125(5):1205-1210

17. Dominguez LJ, Barbagallo M, Lauretani F, Bandinelli S, Bos A, Corsi AM, Simonsick EM, Ferrucci L (2006) Magnesium and muscle performance in older persons: the InCHIANTI study. Am J Clin Nutr 84(2):419-426

18. Brilla LR, Haley TF (1992) Effect of magnesium supplementation on strength training in humans. J Am Coll Nutr 11(3):326-329

19. Zheng L-Z, Wang J-L, Kong L, Huang L, Tian L, Pang Q-Q, Wang X-L, Qin L (2018) Steroid-associated osteonecrosis animal model in rats. J Orthop Transl 13:13-24

20. IoLA R (1996) Guide for the care and used of laboratory animals. National Academies Press

21. Kilkenny C, Browne WJ, Cuthill IC, Emerson M, Altman DG (2010) Improving bioscience research reporting: the 
ARRIVE guidelines for reporting animal research. PLoS Biol 8(6):e1000412. https://doi.org/10.1371/journal.pbio.1000412

22. Slotman G, Fisher CJ Jr, Bone RC, Clemmer TP, Metz CA (1993) Detrimental effects of high-dose methylprednisolone sodium succinate on serum concentrations of hepatic and renal function indicators in severe sepsis and septic shock. The methylprednisolone severe sepsis study group. Crit Care Med 21(2):191-195

23. Nair AB, Jacob S (2016) A simple practice guide for dose conversion between animals and human. J Basic Clin Pharm 7(2):27-31. https://doi.org/10.4103/0976-0105.177703

24. Liu D, Ahmet A, Ward L, Krishnamoorthy P, Mandelcorn ED, Leigh R, Brown JP, Cohen A, Kim H (2013) A practical guide to the monitoring and management of the complications of systemic corticosteroid therapy. Allergy Asthma Clin Immunol 9(1): $1-25$

25. Zheng L-Z, Wang J-L, Xu J-K, Zhang X-T, Liu B-Y, Huang L, Zhang R, Zu H-Y, He X, Mi J (2020) Magnesium and vitamin C supplementation attenuates steroid-associated osteonecrosis in a rat model. Biomaterials 238:119828

26. Kelly FJ, McGrath JA, Goldspink DF, Cullen MJ (1986) A morphological/biochemical study on the actions of corticosteroids on rat skeletal muscle. Muscle Nerve Off J Am Assoc Electrodiagn Med 9(1):1-10

27. Guo A-y, Leung K-s, Qin J-h, Chow SK-h, Cheung W-h (2016) Effect of low-magnitude, high-frequency vibration treatment on retardation of sarcopenia: senescence-accelerated mouse-P8 model. Rejuv Res 19(4):293-302

28. Zhang N, Chow SKH, Leung KS, Lee HH, Cheung WH (2017) An animal model of co-existing sarcopenia and osteoporotic fracture in senescence accelerated mouse prone 8 (SAMP8). Exp Gerontol $97: 1-8$

29. Bloemberg D, Quadrilatero J (2012) Rapid determination of myosin heavy chain expression in rat, mouse, and human skeletal muscle using multicolor immunofluorescence analysis. PLoS ONE 7(4): 35273

30. Luo Y, Li Y, Hu C, Wang J, Qin L, Fu G, Chen S (2020) Gastrin for prevention of steroid-associated osteonecrosis in rats. J Orthop Transl 25:105

31. Huang Z, Fu F, Ye H, Gao H, Tan B, Wang R, Lin N, Qin L, Chen W (2020) Chinese herbal Huo-Gu formula for treatment of steroid-associated osteonecrosis of femoral head: a 14-years follow-up of convalescent SARS patients. J Orthop Transl 23:122

32. Wei Q-S, Hong G-J, Yuan Y-J, Chen Z-Q, Zhang Q-W, He W (2019) Huo Xue Tong Luo capsule, a vasoactive herbal formula prevents progression of asymptomatic osteonecrosis of femoral head: a prospective study. J Orthop Trans1 18:65-73

33. Gröber U, Schmidt J, Kisters K (2015) Magnesium in prevention and therapy. Nutrients 7(9):8199-8226

34. Rolla G, Bucca C, Bugiani M, Oliva A, Branciforte L (1990) Hypomagnesemia in chronic obstructive lung disease: effect of therapy. Magnes Trace Elem 9(3):132-136
35. Chen D, Yang MR, Huang LN, Qiu YW, Li ST (2014) Dexamethasone-induced hyposensitivity to rocuronium in rat diaphragm associated with muscle-fiber transformation. Mol Med Rep 9(2):527-534

36. Decramer M, Stas KJ (1992) Corticosteroid-induced myopathy involving respiratory muscles in patients with chronic obstructive pulmonary disease or asthma. Am Rev Respir Dis 146:800-800

37. Decramer M, Lacquet LM, Fagard R, Rogiers P (1994) Corticosteroids contribute to muscle weakness in chronic airflow obstruction. Am J Respir Crit Care Med 150(1):11-16

38. Castiglioni S, Leidi M, Carpanese E, Maier JA (2013) Extracellular magnesium and in vitro cell differentiation: different behaviour of different cells. Magnes Res 26(1):24-31

39. He K, Liu K, Daviglus ML, Morris SJ, Loria CM, Van Horn L, Jacobs DR Jr, Savage PJ (2006) Magnesium intake and incidence of metabolic syndrome among young adults. Circulation 113(13):1675-1682

40. Belin RJ, He K (2007) Magnesium physiology and pathogenic mechanisms that contribute to the development of the metabolic syndrome. Magnes Res 20(2):107-129

41. Singh R, Rastogi S, Mani U, Seth J, Devi L (1991) Does dietary magnesium modulate blood lipids? Biol Trace Elem Res 30(1):59-64

42. Miljkovic I, Kuipers A, Kuller L, Sheu Y, Bunker C, Patrick A, Wheeler V, Evans R, Zmuda J (2013) Skeletal muscle adiposity is associated with serum lipid and lipoprotein levels in AfroCaribbean men. Obesity 21(9):1900-1907

43. Bredella MA, Gill CM, Gerweck AV, Landa MG, Kumar V, Daley SM, Torriani M, Miller KK (2013) Ectopic and serum lipid levels are positively associated with bone marrow fat in obesity. Radiology 269(2):534-541

44. Braunstein P, De Girolami U (1981) Experimental corticosteroid myopathy. Acta Neuropathol 55(3):167-172

45. Nilwik R, Snijders T, Leenders M, Groen BBL, van Kranenburg J, Verdijk LB, van Loon LJC (2013) The decline in skeletal muscle mass with aging is mainly attributed to a reduction in type II muscle fiber size. Exp Gerontol 48(5):492-498. https://doi.org/ 10.1016/j.exger.2013.02.012

46. Bodine SC, Baehr LM (2014) Skeletal muscle atrophy and the E3 ubiquitin ligases MuRF1 and MAFbx/atrogin-1. Am J PhysiolEndocrinol Metab 307(6):E469-E484

47. Jackman RW, Kandarian SC (2004) The molecular basis of skeletal muscle atrophy. Am J Physiol Cell Physiol 287(4):C834-C843

48. Bertorini TE, Bhattacharya SK, Palmieri GM, Chesney CM, Pifer D, Baker B (1982) Muscle calcium and magnesium content in Duchenne muscular dystrophy. Neurology 32(10):1088-1088 